\begin{tabular}{|c|ccc|c|}
\hline & PORT SAID ENGINEERING RESEARCH JOURNAL \\
\hline
\end{tabular}

\title{
Inter-Area Oscillation Damping With A Near-Optimal Fuzzy Logic Stabilizer
}

\begin{abstract}
K. K. A.El-Serafi ${ }^{1}$ and T. Abdel-Azim ${ }^{1}$

This paper describes a novel power system stabilizer based on fuzzy logic theory (FLPSS) for damping power system oscillations focusing on inter-area modes. In particular, the adaptive fuzzy power system stabilizers using both off-line and on-line training. The determination of the as sociated domains of the fuzzy logic power system stabilizer is obtained by off-line training using a pre-designed optimal controller. Computer simulations for a test power system when subjected to small disturbances under normal and stressed operating conditions are carried out. The dig ital simu lation results show that the proposed controller proves its effectiveness and improves the system damping when compared to a lead-lag and an optimal power system stabilizer controllers.
\end{abstract}

Keywords: Power System Stabilizer, Stability, Fuzzy Logic applications, inter-area modes.

\section{Introduction}

The poorly damped modes of oscillations occur particularly in power systems with longitudinal structure or weak ties. Supplementary excitation controllers as additional feedback signals are used for the last decades to enhance system damping and to improve the dynamic stability of power systems. These controllers are known as power system stabilizers. They have been widely used for many years under various types.

Some of these controllers were designed based on conventional control theory such as PI or lead-lag controllers that cannot provide a total satisfactory response when the operating conditions change widely. These controllers have a main trouble for weekly connected power systems as a trade off will appear at the design process between local-modes and inter-area modes [1].

Optimal controllers which are based on feedback signals from all or some states of the system will guarantee the damping of both local and inter-area modes [2]. These controllers always suffer a lot from on-line iterative solution to Ricatti equation beside the many feedback gain channels [3]. Another type of controllers is the adaptive control, which can do the entire job, but it needs time consumption for real-time system identification $[4,5]$. The simulations associated with steady state stability are quite expensive in computational efforts, so these controllers cannot be used for fast on-line assessments. Additionally, Many controllers such as self-tuning control, sliding-mode control and $\mathrm{H}$-infinity control are a suggested solution to both local and inter-area modes of oscillation but they are not the perfect solution $[6,7]$.

New types of controllers such as rule-based, neural networks and fuzzy logic controllers have been used in many power system applications [8, 9, and 10]. These types of controllers do not require any kind of computational complexity. This point is vital, as a multi-machine system is a large-scale system.

They always need a right off-line design. The present

${ }^{T}$ Department of Electrical Engineering, Faculty of Engineering, Port Said University, Egypt. paper introduces a near optimal novel power system stabilizer based on fuzzy logic theory. This controller has the advantage of its simplicity, systematic design and combination the AVR and PSS functions. The performance of this controller is investigated using a detailed model for a multi-machine test system. The proposed training algorith $m$ is applied on a specific fault at determined operating conditions. It shows the methodology of Appling the off-line training that can be applied to any other faults at different operating conditions. This may yield different fuzzy rules.

\section{Fuzzy Logic Controller}

\subsection{Controller structure}

The proposed controller uses triangular shaped fuzzy sets (as nb:negative big, nm: negative medium, ns: negative small, z:zero, ps:positive small, pm: positive medium and pb: positive big.) Fig. 1, the Max-Min inference method and the center of gravity deffuzification strategy .

Two input signals are suggested, the generator speed deviation signal and generator speed error change. The output signal is the damping control signal. The point wise input for the controllers must be fuzzified (determination of their association to each defined fuzzy set in the domain).

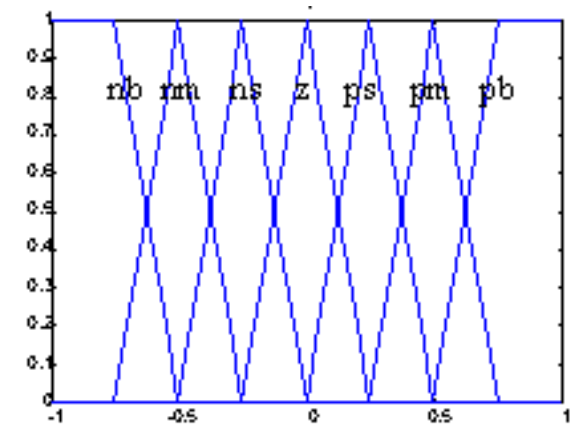

Fig.(1) Classified fuzzy sets. 


\subsection{Controller training}

A simple fuzzy controller based on the experience can damp only local modes. To damp both local and interarea modes of oscillation, the experience is difficult to be obtained. So, the design process needs a systematic method for obtaining the rule base and the domain ranges. The proposed solution of this problem is that a fuzzy controller is to be developed based on the optimal control theory. This is capable to obtain a near optimal fu zzy controller that is characterized by its systematic nature in design.

The main two properties to construct this controller are:

1- Physical domains: They contain their normalized counterparts, the normalization / denormalization scaling factors and the ranges of fuzzy me mbership sets.

2- The rule base defines the relationship between the fuzzy controller inputs and its output in fuzzy manner. Forty-nine rules for the proposed controller are to be extracted.

These requirements for the proposed controller can be achieved by using optimal controllers, which always guarantee the damping of both local and inter-area modes. For the nonlinear system described by the state space equation, the optimal control signal $u$ that minimizes the performance index is a linear function in terms of the system state variable $\mathrm{x}$ as

$\mathrm{Q}$ and $\mathrm{R}$ are the weighting matrices, $\mathrm{K}$ is the feedback gain matrix for the output $\mathrm{u}$ and $\mathrm{P}$ is

$$
\begin{aligned}
& \mathrm{J}=\frac{1}{2} \int_{0}^{\infty}\left(\mathrm{x}^{\mathrm{T}} \mathrm{Qx}+\mathrm{u}^{\mathrm{T}} \mathrm{Ru}\right) \mathrm{dt} \\
& \mathrm{u}=\mathrm{Kx}=-\mathrm{R}^{-1} \mathrm{~B}^{\mathrm{T}} \mathrm{Px}
\end{aligned}
$$

the solution of the linear matrix Riccati equation:

$$
\mathrm{A}^{\mathrm{T}} \mathrm{P}+\mathrm{PA}-\mathrm{PBR}^{-1} \mathrm{~B}^{\mathrm{T}} \mathrm{P}+\mathrm{Q}=0
$$

A systematic design for the optimal controller can be obtained from [11]. Physical domains can be calculated from the generated data for simulation by the optimal controller assuming different disturbances .

For the rule base, the relationship between the fuzzy controller inputs and its output can be extracted from this algorith $m$.

Step1: simulate the optimal controller

Step 2: Save each sample value of $(\Delta \omega$, change in $\Delta \omega$ , Upss)

Step 3: At each sample time t:

$\Delta \omega \in$ the class with $\max$ membership among ( $\omega \_n b$, $\left.\omega \_n m, \omega \_n s, \omega \_z, \omega \_p s, \omega \_p m, \omega \_p b\right)$

so at sample time $\Delta \omega$ is $\omega_{-} 1$

change in $\Delta \omega \in$ the class with max membership among ( $d \omega \_n b, d \omega \_n m, d \omega \_n s, d \omega \_z, d \omega \_p s, d \omega \_p m, d \omega \_p b$ )

so at sample time $\mathrm{t}, \Delta \omega$ is $\mathrm{d} \omega_{-} 1$

This will form the contents of the rule-antecedent (If-part of a rule) so at sample time $\mathrm{t}$, Upss is $\mathrm{u}_{-} 1$

This The contents of the rule-consequent (then-part of the rule)

And a total rule can be formed as:

From (a), (b) and (c) the rule

"If $\Delta \omega$ is $\omega_{-} 1$ and change in $\Delta \omega$ is d $\omega_{-} 1$ then Upss is u_1"

Note: After generation of rules, only small amount of samples can violate the rule base table. These samples are denied according to the results Table.1.

\section{Multimachine Test System}

The single-line diagram of the two-area, 4-machine test system as shown in Fig. 2 is used to examine both local and inter-area oscillations control problem. This system is created es pecially for the analysis and study of the inter-area oscillation problem [1].

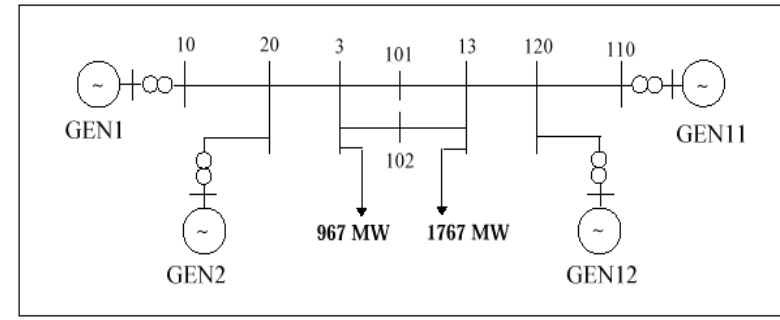

Fig.(2): Two area multi-machine test system.

As shown in the single-line diagram there are four generators, GEN1, GEN2, GEN11 and GEN12, and four $20 / 230 \mathrm{kV}$ step-up transformers. There are two loads in the system at buses 3 and 13. The transformer and line impedances for the system are given in Appendix (A). This system exhibits three electromechanical modes of oscillations. One inter-area mode in which the generating units in one area oscillates against those in the other area. The frequency of this mode varies from $(0.35-0.75)$ $\mathrm{Hz}$ depending on operating conditions. Two local modes which represent oscillations between the generating units within each area. The frequency of the local modes is around $1.3 \mathrm{~Hz}$. The loads are modeled as constant impedances. A $400 \mathrm{MW}$ power transfer from area \#1 to area\#2 is the main case study that is very stressed operating point. One set of FLC controllers is used for each area. This set of controllers includes one FLC for the area and one FLC for each generator within the area. The test contains two areas. A comparison between the results of a lead-lag, optimal and fuzzy controllers due to different disturbances is presented. A comparison between the resultant performance of the optimal and fuzzy controller is needed. So the authors compared the resultant values for the relation (1) for both controllers. For instance, when a disturbance of $5 \%$ increase in rotor speed occurs at one of system generators, Figs 3-6 depictthe performance of FLC, lead-lag, and optimal controller. The results show that the fuzzy controller perfor- 
Table.1 Rule base of FLPSS extracted from optimal control simulation

\begin{tabular}{|c|c|c|c|c|c|c|c|}
\hline \multirow{2}{*}{ Speed Deviation } & \multicolumn{7}{|c|}{ Speed Deviation Change } \\
\hline & $\mathrm{d} \omega \_n b$ & $\mathrm{~d} \omega \_n \mathrm{~m}$ & $\mathrm{~d} \omega \_n s$ & $\mathrm{~d} \omega \_z$ & $\mathrm{~d} \omega \_p s$ & $\mathrm{~d} \omega \_\mathrm{pm}$ & $\mathrm{d} \omega \_p b$ \\
\hline$\omega \_n b$ & u_nb & u_nb & u_nb & u_nb & u_nm & u_ps & $\mathrm{u} \_\mathrm{Z}$ \\
\hline$\omega \_n m$ & u_nb & u_nm & u_nm & u_nm & u_ns & u_z & u_ps \\
\hline$\omega \_n s$ & u_nb & u_nm & u_ns & u_ns & u_z & u_ps & u_pm \\
\hline$\omega \_Z$ & u_nb & u_nm & u_ns & u_Z & u_ps & u_pm & $\mathrm{u} \_\mathrm{pb}$ \\
\hline$\omega \_p s$ & u_nm & u_ns & u_z & u_ps & u_ps & u_pm & $\mathrm{u} \_\mathrm{pb}$ \\
\hline$\omega_{-} \mathrm{pm}$ & u_ns & $u_{-} z$ & u_ps & u_pm & u_pm & $\mathrm{u} \_\mathrm{pb}$ & $\mathrm{u} \_\mathrm{pb}$ \\
\hline$\omega \_\mathrm{pb}$ & u_z & u_ps & u_pm & u_pb & u_pb & u_pb & $\mathrm{u} \_\mathrm{pb}$ \\
\hline
\end{tabular}

mance index ranges from $8 \%-14 \%$ larger than the optimal controller performance index for different disturbances.

\section{Conclusions}

The performed study work in this research involves a fuzzy logic controller, which is built based on the data generated by an optimal controller. A systematic generation of a fuzzy logic controller rule base and input-output domain ranges has been investigated and tested. It has been found that it provides a more robust control over a

Large excursion of the operating points versus the lead-lag stabilizer and very near from the optimal controller performance.

Most of the previous control methods either are not working sufficiently under whole range of operating conditions or they need complicated calculations, as they require the exact model. A decrease in calculations due to the fuzzy controller is a fact. The result of the proposed controller has indicated damping increase and stability enhancement of both local and inter-area modes. As a future work, the off-line- training algorith $m$ is recommended to be applied to a large numbers of operating conditions, a large number of scenarios and different clearing times. This is to examine the adequacy of fuzzy rules that may change with varying scenarios.

\section{REFRENCES}

[1] D. P. Ke and C. Y. Chung,"An Inter-Area Mode Oriented Pole-Shifting Method With Coordination of Control Efforts for Robust Tuning of Power Oscillation Damping Controllers", IEEE Transactions on Power Systems, Vol.27, No.3, pp.1422-1432, March 2012.

[2] T .T. Lie, G .J. Li, G. B. Shrestha, and K. L. Lo ,” Coordinated decentralized optimal control of interarea oscillations in power systems", 1998 International Conference on Energy Management and Power Delivery, Vol.1, pp.97-102, August 1998.

[3] M. E. Aboul-Ela, "Two-Level Control Strategy for Damping Power System Oscillations", PHD. Thesis, Suez Canal University, May 1994.
[4] G. Swann and S. Kamalasadan, "A Novel SystemCentric Intelligent Adaptive Control Architecture for Damping Inter-Area Mode Oscillations in Power System", 2010 IEEE Industry Applications Society Annual Meeting (IAS), pp.1-7, October 2010.

[5] J. Ma, T. Wand, Z. Wang, J. S. Thorp, "Adaptive Damping Control of Inter-A rea Oscillations Based on Federated Kalman Filter Using Wide Area Signals", IEEE Transactions on Power Systems, Vol. 28, No.2, pp. 1627-1635, April 2013.

[6] Jianying Gong, Rong Xie, Weiguo Liu , and Shuanqin Xie, "H-Infinity Controller Design of the Synchronous Generator", 2008 International Conference on Intelligent Computation Technology and Automation (ICICTA), Vol.1, pp. 370 - 374, October 2008

[7] Sheng-Kuan Wang, "A Novel Objective Function and Algorithm for Optimal PSS Parameter Design in a Multi-Machine Power System", IEEE Transactions on Power Systems , Vol. 28, No.1, 531pp.522-, June 2013.

[8] P.J. Costa Branco, “The Application of Fuzzy Logic in Automatic Modelling of Electromechanical Systems", Fuzzy Sets and Systems, Elsevier Science, Jan. 1999.

[9] A. Hariri, O.P. Malik, "Fuzzy logic power system stabilizer based on genetically optimized adaptive network", Elsevier Science, Jan 2000.

[10] K. Gowrishankar and M. Khan, "MATLAB simulink model of fuzzy logic controller with PSS and its performance analysis", 2012 International Conference on Advances in Engineering, Science and Management (ICAESM), Vol.1, pp.541-550, March 2012

[11] G. P. Chen, Y.Q. Qin , G.Y. Xu and O. P. Malik,"Optimization Technique for The Design of a Linear Optimal Power System Stabilizer", IEEE Tran sactions on Energy Conversion, Vol.7, No.3, pp.453459, Septe mber 1992. 

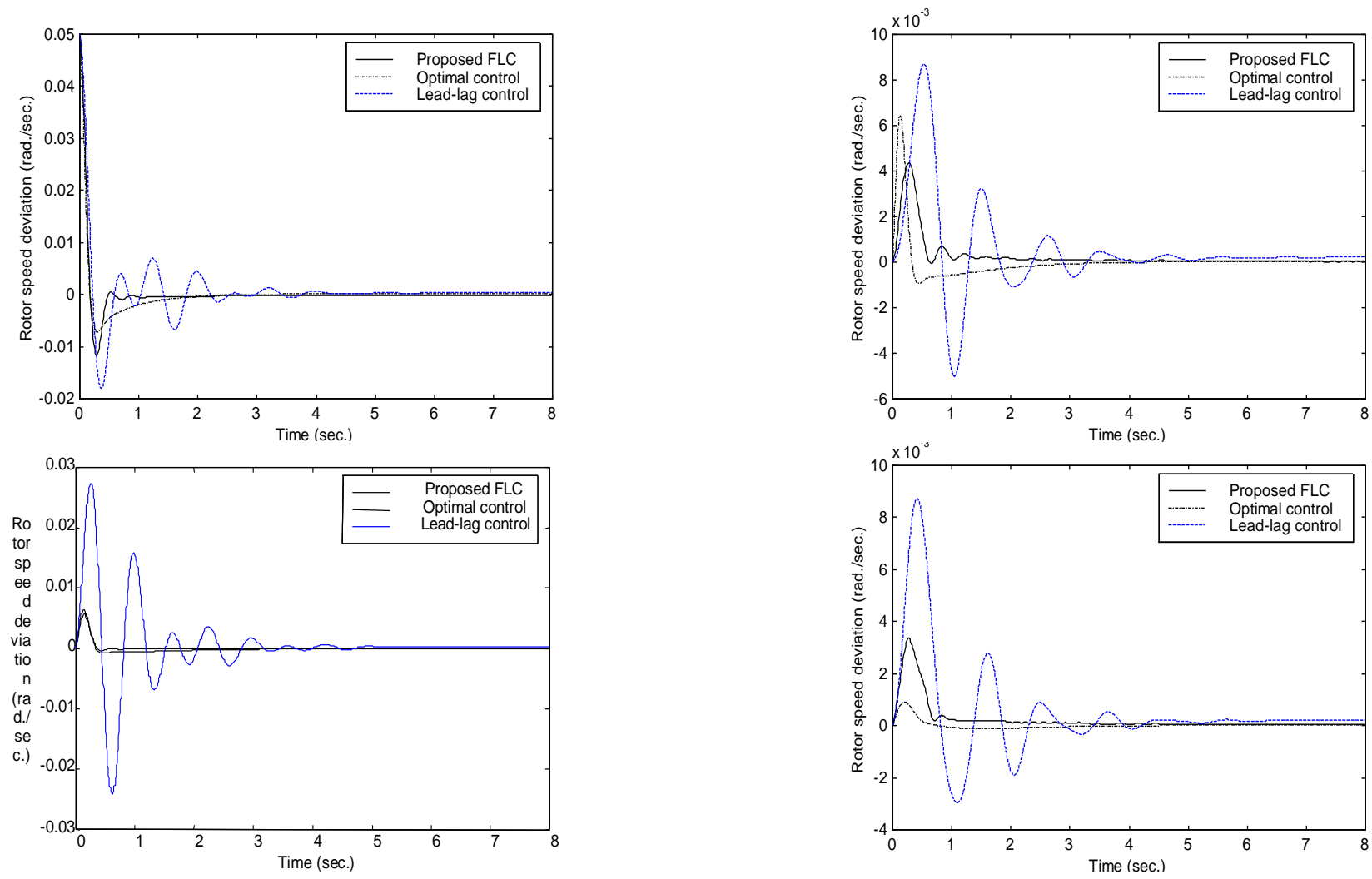

Fig.(3) : Case1 An increase of $5 \%$ in rotor s peed at Gen1
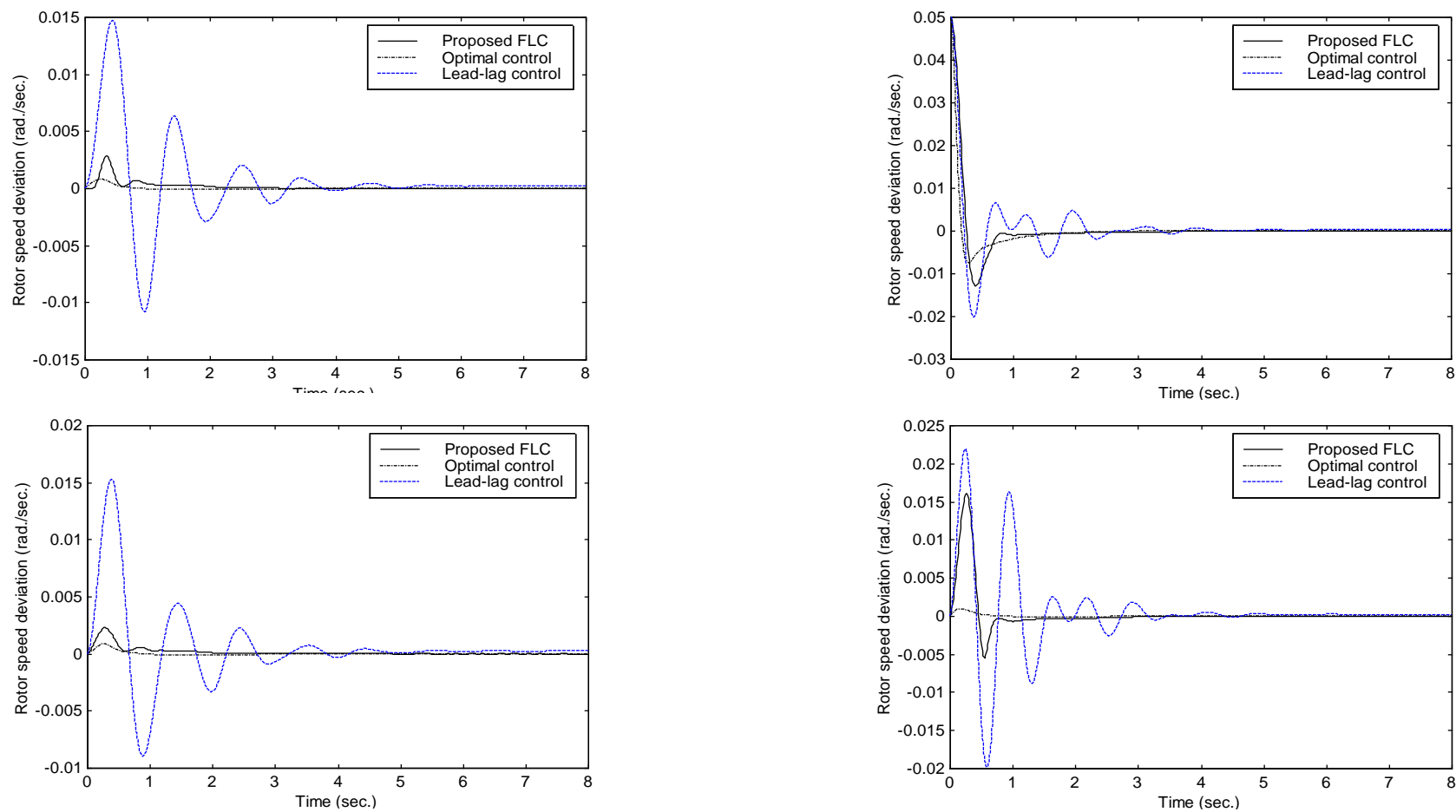

Fig.(4) :Case2 An increase of 5\% in rotor speed at Gen2. 

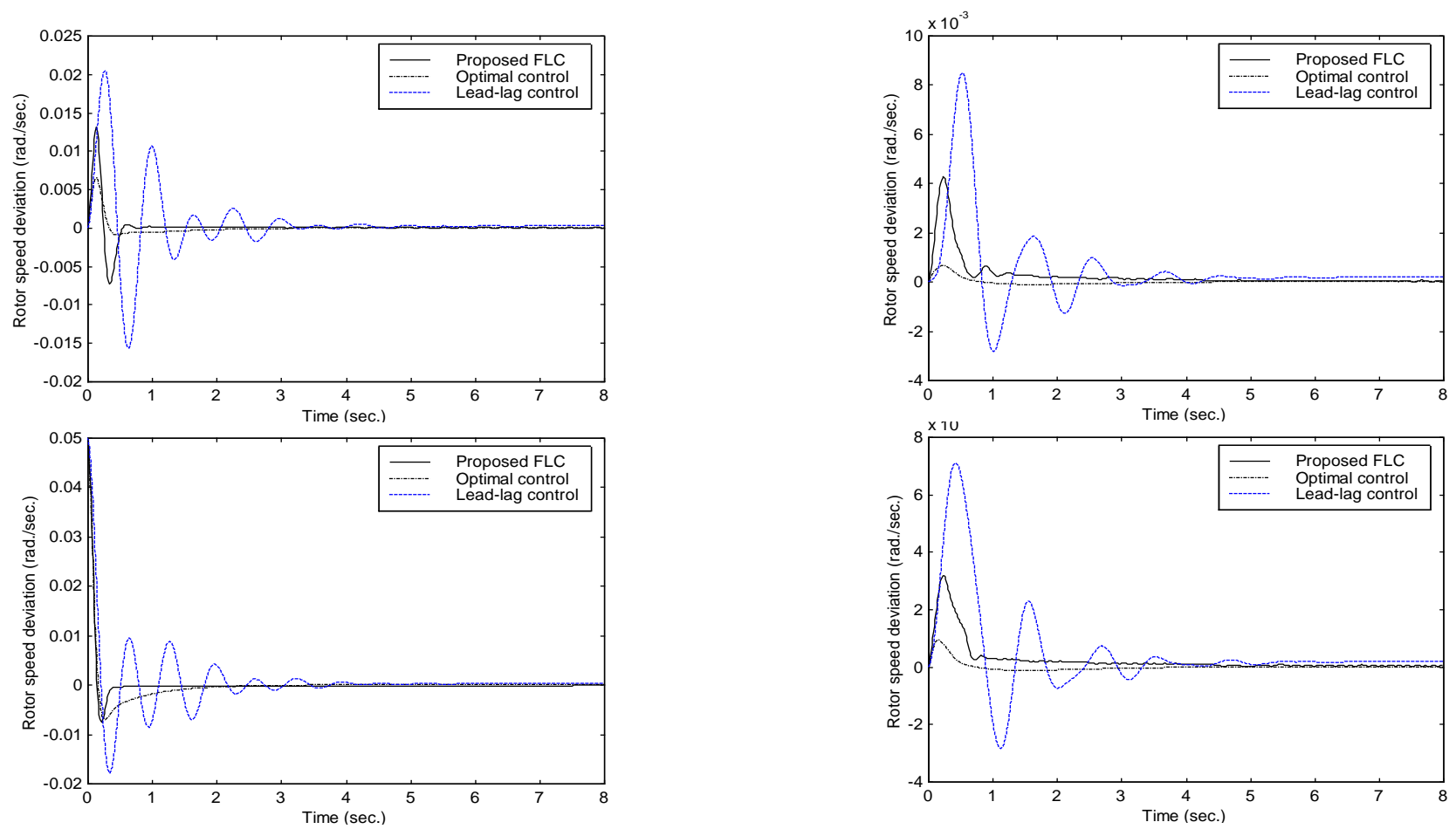

Fig.(5) : Case3 An increase of $5 \%$ in rotor s peed at Gen11.
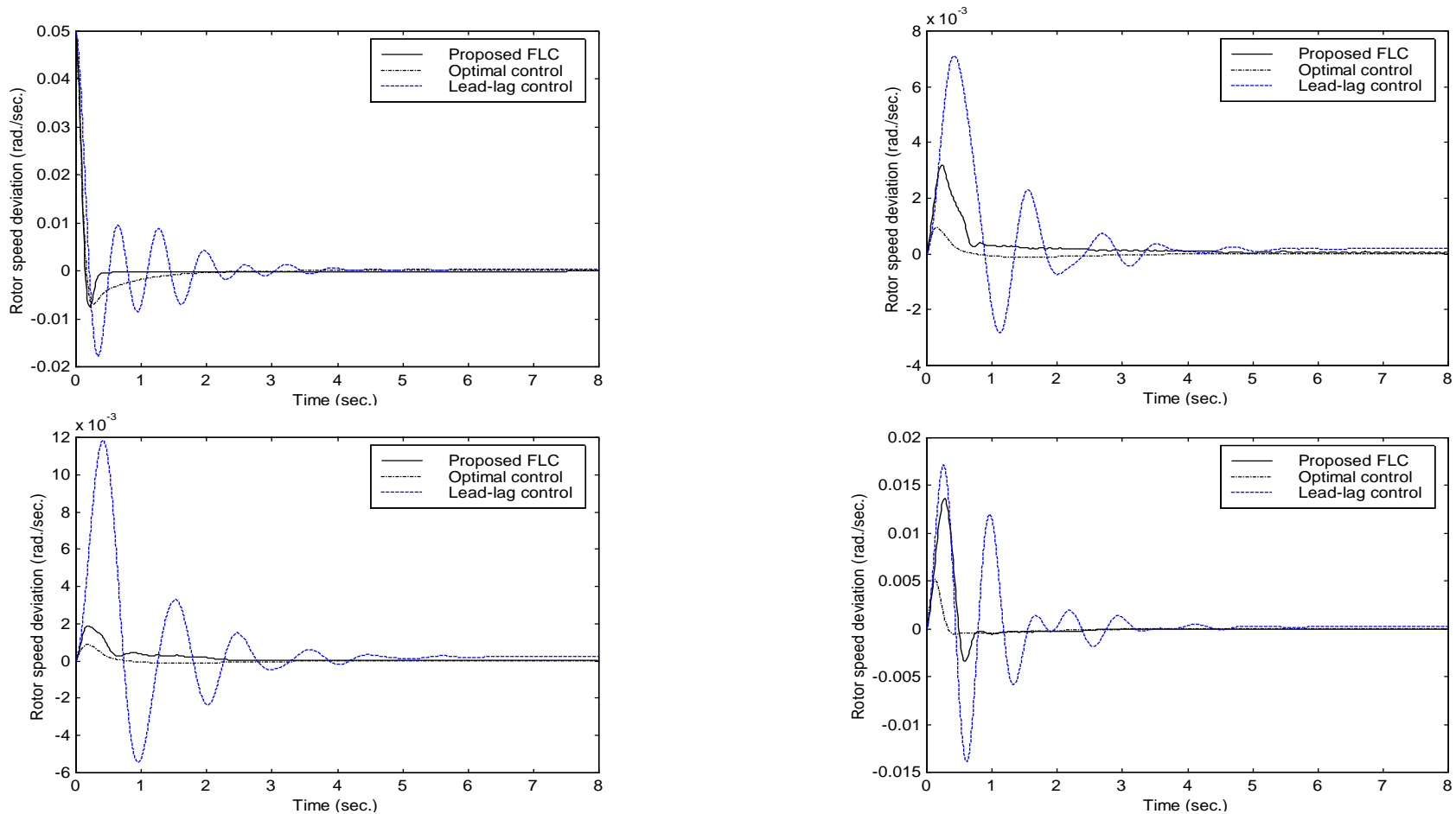

Fig (6): Case4 An increase of $5 \%$ in rotor s peed at Gen12. 


\section{Appendix A (Machine Test System Data)}

\section{A.1 the Generator Parameters}

$\mathrm{Xd}=1.8 \mathrm{Xq}=1.7 \mathrm{Xl}=0.2 \mathrm{Xd}^{`}=0.3 \mathrm{Xq}^{`}=0.55 \mathrm{Xd}=0.25$ $\mathrm{Xq}$ “ $=0.25 \mathrm{Ra}=0.0025 \mathrm{~T}$ d $0=8 \mathrm{~s}$ T'q0 $=0.4 \mathrm{~s}$ T“d $0=0.03$ s T“q0 $=0.05$ s Asat=0.015 Bsat=9.6 H=6.5 (G1 \& G2) $\mathrm{H}=6.175$ (G11 \& G12) KD=0

\section{A.2 Impedance Data for 4-Machine System}

\begin{tabular}{|c|c|c|c|c|}
\hline $\begin{array}{c}\text { From } \\
\text { Bus }\end{array}$ & $\begin{array}{c}\text { To } \\
\text { Bus }\end{array}$ & $\mathrm{R}(\mathrm{pu})$ & $\mathrm{X}(\mathrm{pu})$ & $\mathrm{B} / 2(\mathrm{pu})$ \\
\hline GEN1 & 10 & 0.0 & 0.0167 & 0.0 \\
\hline GEN2 & 20 & 0.0 & 0.0167 & 0.0 \\
\hline GEN11 & 110 & 0.0 & 0.0167 & 0.0 \\
\hline GEN12 & 120 & 0.0 & 0.0167 & 0.0 \\
\hline 10 & 20 & 0.0025 & 0.025 & 0.021875 \\
\hline 20 & 3 & 0.001 & 0.01 & 0.00875 \\
\hline 3 & 101 & 0.011 & 0.11 & 0.09625 \\
\hline 3 & 102 & 0.011 & 0.11 & 0.09625 \\
\hline 101 & 13 & 0.011 & 0.11 & 0.09625 \\
\hline 102 & 13 & 0.011 & 0.11 & 0.09625 \\
\hline 120 & 110 & 0.0025 & 0.025 & 0.021875 \\
\hline 13 & 120 & 0.001 & 0.01 & 0.00875 \\
\hline
\end{tabular}

A.3 Thyristor Exciter with a High Transient Gain $K_{A}=200 T_{R}=0.01$ Sec.

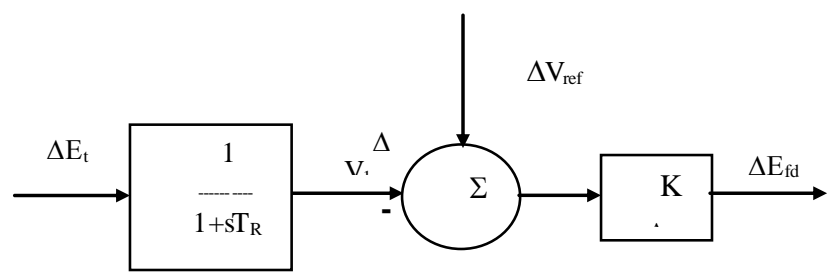

\section{A.4 Two Stage Lead-Lag Power System Stabilizer} Data

stab $=200 T_{w}=10$ Sec. $T_{1}=0.05$ Sec. $T_{2}=0.02$ Sec. $T_{3}=3$ Sec. $\mathrm{T}_{4}=5.4 \mathrm{Sec}$

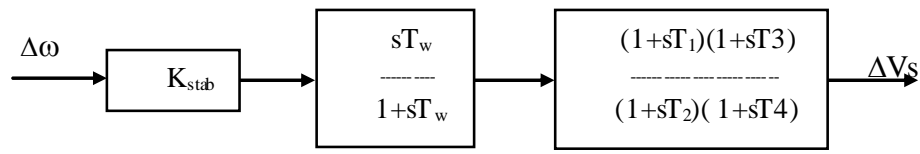

